HU-EP-06/20

hep-th/0608013

\title{
Instantons and Emergent Geometry
}

\author{
Hyun Seok Yang* \\ Institut für Physik, Humboldt Universität zu Berlin, Newtonstraße 15, D-12489 Berlin, Germany
}

(Dated: April 19, 2021)

\begin{abstract}
We show that self-dual electromagnetism in noncommutative spacetime is equivalent to self-dual Einstein gravity.

PACS numbers: 11.10.Nx, 02.40.Gh, 11.25.Tq

Keywords: Emergent geometry, Noncommutative instanton, Gravitational instanton, Twistor space
\end{abstract}

\section{INTRODUCTION}

Gravity is a mysterious force; we still don't know what gravity is. But recent developments from string and $\mathrm{M}$ theories gradually reveal a remarkable and radical new picture about gravity (for recent reviews, see, for example, [1, 2]): Gravity may be a collective phenomenon emergent from gauge fields. That is, the spin-two graviton might arise as a composite of two spin-one gauge bosons.

Although the emergent gravity is a fascinating approach suggesting that gravity itself may not be "fundamental physics", it is not easy to realize it from ordinary quantum field theories due to a general no-go theorem known as the Weinberg-Witten theorem [3], stating that an interacting graviton cannot emerge from an ordinary quantum field theory in the same spacetime. One has to notice, however, that Weinberg and Witten introduced two basic assumptions to prove this theorem. The first hidden assumption is that gravitons and gauge fields live in the same spacetime. The second assumption is the existence of a Lorentz-covariant stress-energy tensor. Thus, to realize the emergent gravity, one has to relax one of the assumptions in some ways.

One also has to recall that gravity has no local gaugeinvariant degrees of freedom. So one cannot start with a theory containing local gauge-invariant operators to generate Einstein gravity as an emergent phenomenon in the same spacetime.

The clue to relax the first assumption comes from the holographic principle [4]: Gravitons live in a higher dimensional spacetime than gauge fields. In this description known as the anti-de Sitter space and conformal field theory (AdS/CFT) duality [5], gravity in higher dimensions is an emergent phenomenon arising from particle interactions in a gravityless, lower-dimensional world. The bulk geometry, i.e., AdS space, has to contain a holographic screen on which the boundary theory, typically super Yang-Mills theory, is defined. Since the Yang-Mills theory contains many local gauge-invariant observables and the diffeomorphism only appears in the bulk, the AdS/CFT duality does not provide any information on lower-dimensional gravity in the same spacetime where the boundary theory is defined.
One may try to relax the second assumption instead. This assumption can be relaxed, for example, by introducing a symplectic structure of spacetime - noncommutative (NC) spacetime:

$$
\left[y^{\mu}, y^{\nu}\right]_{\star}=i \theta^{\mu \nu} .
$$

A field theory defined on the $\mathrm{NC}$ spacetime preserves neither locality nor Lorentz invariance. An important fact is that translations in $\mathrm{NC}$ directions are basically gauge transformations, i.e.,

$$
e^{i k \cdot y} \star f(y) \star e^{-i k \cdot y}=f(y+\theta \cdot k) .
$$

This immediately implies that there are no local gaugeinvariant observables in NC gauge theory [6]. These properties are precisely those of gravity. This was the motivation in 7] to explore the relation between $\mathrm{NC}$ field theory and gravity.

Recently it was shown in [8, 9] that gravitational instantons in Einstein gravity are equivalent to $U(1)$ instantons in NC gauge theory, so illuminating the remarkable correspondence between NC field theory and gravity at least for their self-dual sectors.

In this paper we will generalize the results in [8, 9], showing that self-dual electromagnetism in NC spacetime is equivalent to self-dual Einstein gravity. Although the equivalence between $\mathrm{NC}$ instantons and gravitational instantons was formally shown in 8, 9], an explicit example was shown only for the Eguchi-Hanson metric and a single NC instanton. Furthermore it is a highly nontrivial problem to find multi-instanton solutions even in the gauge theory. Therefore one might expect that it will be very difficult to explicitly find a map that sends a generic self-dual gauge field configuration of $\mathrm{NC}$ gauge theory to a metric which is a solution of self-dual Einstein equations. It is thus quite surprising that there is an elegant formulation to realize the equivalence between selfdual NC electromagnetism and self-dual Einstein gravity, where it is not difficult to find explicit nontrivial solutions showing that the asymptotically locally Euclidean (ALE) and asymptotically locally flat (ALF) spaces 10] as well as the real heaven [11] are indeed solutions of $\mathrm{NC}$ electromagnetism.

This formulation is essentially based on Eq.(2) stating that translations are an inner automorphism of NC algebra (11) or, in its infinitesimal form, derivations are inner, 
i.e.,

$$
-i\left[\left(\theta^{-1}\right)_{\mu \nu} y^{\nu}, f(y)\right]_{\star}=\partial_{\mu} f(y) .
$$

In the presence of gauge fields, the coordinates $y^{\mu}$ should be promoted to the covariant coordinates defined by

$$
x^{\mu}(y) \equiv y^{\mu}+\theta^{\mu \nu} \widehat{A}_{\nu}(y)
$$

in order for star multiplications to preserve the gauge covariance [12]. The inner derivations (3) are accordingly covariantized too as follows

$$
\begin{aligned}
\operatorname{ad}_{D_{\mu}}[f] & \equiv-i\left[D_{\mu}(y), f(y)\right]_{\star}=\theta^{a b} \frac{\partial D_{\mu}}{\partial y^{a}} \frac{\partial f}{\partial y^{b}}+\cdots \\
& \equiv V_{\mu}^{a}(y) \partial_{a} f(y)+\mathcal{O}\left(\theta^{3}\right)
\end{aligned}
$$

where $D_{\mu}(y) \equiv\left(\theta^{-1}\right)_{\mu \nu} x^{\nu}(y)$. It turns out that the vector fields $V_{\mu}(y) \equiv V_{\mu}^{a}(y) \partial_{a}$ form an orthogonal frame and hence define vielbeins of a gravitational metric. This intrinsic property in NC geometry will be combined with the rigorously established result in 13, 14, 15] to conclude that self-dual NC electromagnetism is equivalent to self-dual Einstein gravity, which will be done in Section 3.

Self-dual gauge fields in NC spacetime have been shown in [8, 9] to describe hyper-Kähler manifolds and every hyper-Kähler manifold has an associated twistor space [16]. Therefore we expect that self-dual NC electromagnetism may be related to a twistor space describing curved self-dual spacetime. We will show that this structure is beautifully realized through the Darboux theorem, where all the information of self-dual NC gauge fields can be encoded in the holomorphic structure of the twistor space. This construction also clarifies the nature of gravity emerging from $\mathrm{NC}$ gauge fields; the gauge fields act as a deformation of the Kähler structure of self-dual 4manifold or the complex structure of twistor space. In this way gauge fields in NC spacetime manifest themselves as a deformation of background geometry, which is also compatible with the picture in Eq. (5). Thus we should think of the twistor space as already incorporating the backreaction of NC instantons. This picture is very similar to that in 17] where placing D1-branes (as instantons in gauge theory) in twistor space is interpreted as blowing up points in four dimensions dubbed as spacetime foams and the Kähler blowups in four dimensions are encoded in the twistor space as the backreaction of the D1-branes.

A brief outline of the paper is the following. In Section 2, the equivalence between $\mathrm{NC} U(1)$ instantons and gravitational instantons is made more precise giving a nice geometrical picture in terms of the twistor geometry [18]. The beautiful geometrical structures of twistor geometry also allow us to clarify the nature of gravity emerging from NC gauge fields. In Section 3, we apply Eq.(5) to self-dual NC gauge fields to show the equivalence between self-dual electromagnetism in NC spacetime and self-dual Einstein gravity, using the rigorously established result in [13, 14, 15]. In Section 4, we add some remarks about our results.

\section{NONCOMMUTATIVE INSTANTONS AND TWISTOR SPACE}

Let us consider electromagnetism in the NC spacetime defined by Eq.(1). The action for the NC $U(1)$ gauge theory in flat Euclidean $\mathbf{R}^{4}$ is given by

$$
\widehat{S}_{\mathrm{NC}}=\frac{1}{4} \int d^{4} y \widehat{F}_{\mu \nu} \star \widehat{F}^{\mu \nu},
$$

where NC electromagnetic fields are defined by

$$
\widehat{F}_{\mu \nu}=\partial_{\mu} \widehat{A}_{\nu}-\partial_{\nu} \widehat{A}_{\mu}-i\left[\widehat{A}_{\mu}, \widehat{A}_{\nu}\right]_{\star} .
$$

Contrary to ordinary electromagnetism, the NC $U(1)$ gauge theory admits non-singular instanton solutions satisfying the NC self-duality equation [19],

$$
\widehat{F}_{\mu \nu}(y)= \pm \frac{1}{2} \varepsilon_{\mu \nu \lambda \sigma} \widehat{F}_{\lambda \sigma}(y) .
$$

The NC gauge theory has an equivalent dual description through the so-called Seiberg-Witten (SW) map in terms of ordinary gauge theory on commutative spacetime [20]. The SW map is a map between gauge orbit spaces of commutative and $\mathrm{NC}$ gauge fields. We will mainly be confined to semi-classical limit, say $\mathcal{O}(\theta)$, which means slowly varying fields, $\sqrt{\kappa}\left|\frac{\partial F}{F}\right| \ll 1$, in the sense keeping field strengths (without restriction on their size) but not their derivatives. This is precisely the limit taking only the leading term in Eq.(15), so ignoring derivative corrections which start from $\mathcal{O}\left(\theta^{3}\right)$. (See 21] for derivative corrections of the SW map.) The exact SW map [22, 23] in this limit is given by

$$
\begin{aligned}
& \widehat{F}_{\mu \nu}(y)=\left(\frac{1}{1+F \theta} F\right)_{\mu \nu}(x), \\
& d^{4} y=d^{4} x \sqrt{\operatorname{det}(1+F \theta)}(x),
\end{aligned}
$$

where $x^{\mu}$ 's are the covariant coordinates defined by Eq.(44). Applying the maps (9) and (10) to the action (6), one can get the commutative nonlinear electrodynamics 23, 24] equivalent to Eq. (6)

$$
S_{\mathrm{C}}=\frac{1}{4} \int d^{4} x \sqrt{\operatorname{det} g} \mathrm{~g}^{\mu \lambda} \mathrm{g}^{\sigma \nu} F_{\mu \nu} F_{\lambda \sigma},
$$

where we introduced an "effective metric" induced by the dynamical gauge fields as

$$
\mathrm{g}_{\mu \nu}=\delta_{\mu \nu}+(F \theta)_{\mu \nu}, \quad\left(\mathrm{g}^{-1}\right)^{\mu \nu} \equiv \mathrm{g}^{\mu \nu}=\left(\frac{1}{1+F \theta}\right)^{\mu \nu} .
$$

It was shown in [8] that the self-duality equation for the action $S_{\mathrm{C}}$ is given by

$$
\mathbf{F}_{\mu \nu}(x)= \pm \frac{1}{2} \varepsilon_{\mu \nu \lambda \sigma} \mathbf{F}_{\lambda \sigma}(x),
$$


where

$$
\mathbf{F}_{\mu \nu}(x) \equiv\left(\mathrm{g}^{-1} F\right)_{\mu \nu}(x)
$$

Note that Eq. (13) is nothing but the exact SW map (9) of the NC self-duality equation (8). It turned out [8, 9] that Eq.13) describes gravitational instantons obeying the self-dual equations [25]

$$
R_{a b c d}= \pm \frac{1}{2} \varepsilon_{a b e f} R^{e f}{ }_{c d}
$$

where $R_{a b c d}$ is a curvature tensor. Interestingly, Eq. (13) can be rewritten [21] as the self-duality in a curved space described by the metric $\mathrm{g}_{\mu \nu}$

$$
F_{\mu \nu}(x)= \pm \frac{1}{2} \frac{\varepsilon^{\lambda \sigma \rho \tau}}{\sqrt{\operatorname{det} g}} \mathrm{~g}_{\mu \lambda} \mathrm{g}_{\nu \sigma} F_{\rho \tau}(x) .
$$

It should be remarked, however, that the self-duality in (16) cannot be interpreted as a usual self-duality equation in a fixed background since the four-dimensional metric used to define Eq.(16) depends in turn on the $U(1)$ gauge fields.

$\mathrm{NC}$ instantons turn out to have a rich connection with twistor geometry [18]. We will newly derive the key results in [8, 9] closely following the paper [26] on $N=2$ strings which will clarify the nature of gravity emerging from NC gauge fields. To proceed in that direction, let us consider the line element (where we follow the notations in [8, 9])

$$
d s^{2}=\widetilde{\mathrm{g}}_{\mu \nu} d x^{\mu} d x^{\nu}=\widetilde{\sigma}_{\mu} \otimes \widetilde{\sigma}_{\mu}
$$

where $\mathrm{g}_{\mu \nu}=1 / 2\left(\delta_{\mu \nu}+\widetilde{\mathrm{g}}_{\mu \nu}\right)$. We also introduce the triple of Kähler forms as follows,

$$
\widetilde{\omega}^{a}=\frac{1}{2} \eta_{\mu \nu}^{a} \widetilde{\sigma}^{\mu} \wedge \widetilde{\sigma}^{\nu}
$$

It is easy to check [9] that $\widetilde{\sigma}_{1} \wedge \widetilde{\sigma}_{2} \wedge \widetilde{\sigma}_{3} \wedge \widetilde{\sigma}_{4}=d^{4} x$, say, $\sqrt{\operatorname{det} \widetilde{g}_{\mu \nu}}=1$ and

$$
\begin{aligned}
& \omega \equiv \widetilde{\omega}^{2}+i \widetilde{\omega}^{1}=d z_{1} \wedge d z_{2}, \bar{\omega} \equiv \widetilde{\omega}^{2}-i \widetilde{\omega}^{1}=d \bar{z}_{1} \wedge d \bar{z}_{2} \\
& \Omega \equiv-\widetilde{\omega}^{3}=\frac{i}{2}\left(d z_{1} \wedge d \bar{z}_{1}+d z_{2} \wedge d \bar{z}_{2}\right)+\theta F
\end{aligned}
$$

It is obvious that $d \widetilde{\omega}^{a}=0, \forall a$ because of the Bianchi identity, $d F=0$. This means that the metric $\widetilde{\mathrm{g}}_{\mu \nu}$ is hyper-Kähler [9], which is an equivalent statement as Ricci-flat Kähler in four dimensions. Therefore the metric $\widetilde{\mathrm{g}}_{\mu \nu}$ is a gravitational instanton [25]. Eq.(19) clearly shows how dynamical gauge fields living in NC spacetime deform the Kähler structure and thus induce a deformation of background geometry through gravitational instantons, thus realizing the emergent geometry.

The deformation of symplectic (or Kähler) structure on $\mathbf{R}^{4}$ due to the fluctuation of gauge fields can be more clarified by the following construction. Let us consider a deformation of the holomorphic (2,0)-form $\omega=d z_{1} \wedge d z_{2}$ as follows

$$
\Psi(t)=\omega+i t \Omega+\frac{t^{2}}{4} \bar{\omega}
$$

where the parameter $t$ takes values in $\mathbf{P}^{1}=\mathbf{S}^{2}$. One can easily see that $d \Psi(t)=0$ if and only if $d F=0$ and

$$
\Psi(t) \wedge \Psi(t)=0
$$

since Eq.21 is equivalent to the instanton equation $F_{\mu \nu}^{+}=1 / 4(F \widetilde{F}) \theta_{\mu \nu}^{+}$[8, 9]. Since the two-form $\Psi(t)$ is closed and degenerate, the Darboux theorem asserts that one can find a $t$-dependent map $\left(z_{1}, z_{2}\right) \rightarrow$ $\left(Z_{1}\left(t ; z_{i}, \bar{z}_{i}\right), Z_{2}\left(t ; z_{i}, \bar{z}_{i}\right)\right)$ such that

$$
\Psi(t)=d Z_{1}\left(t ; z_{i}, \bar{z}_{i}\right) \wedge d Z_{2}\left(t ; z_{i}, \bar{z}_{i}\right)
$$

When $t$ is small, one can solve (22) by expanding $Z_{i}(t ; z, \bar{z})$ in powers of $t$ as

$$
Z_{i}(t ; z, \bar{z})=z_{i}+\sum_{n=1}^{\infty} \frac{t^{n}}{n} p_{n}^{i}(z, \bar{z}) .
$$

By substituting this into Eq.(20), one gets at $\mathcal{O}(t)$

$$
\begin{aligned}
& \partial_{z_{i}} p_{1}^{i}=0, \\
& \epsilon_{i k} \partial_{\bar{z}_{j}} p_{1}^{k} d z^{i} \wedge d \bar{z}^{j}=i \Omega
\end{aligned}
$$

where the fact was used that $\Omega$ is a $(1,1)$-form. Eq. (24) can be solved by setting $p_{1}^{i}=1 / 2 \epsilon^{i j} \partial_{z_{j}} K$ and then $\Omega=$ $i / 2 \partial_{i} \bar{\partial}_{j} K d z^{i} \wedge d \bar{z}^{j}$. The real-valued smooth function $K$ is the Kähler potential of $U(1)$ instantons in [8, 9]. In terms of this Kähler two-form $\Omega$, Eq.(21) reduces to the complex Monge-Ampère or the Plebański equation [27]

$$
\Omega \wedge \Omega=\frac{1}{2} \omega \wedge \bar{\omega},
$$

that is, $\operatorname{det}\left(\partial_{i} \bar{\partial}_{j} K\right)=1$.

When $t$ is large, one can introduce another Darboux coordinates $\widetilde{Z}_{i}\left(t ; z_{i}, \bar{z}_{i}\right)$ such that

$$
\Psi(t)=t^{2} d \widetilde{Z}_{1}\left(t ; z_{i}, \bar{z}_{i}\right) \wedge d \widetilde{Z}_{2}\left(t ; z_{i}, \bar{z}_{i}\right)
$$

with expansion

$$
\widetilde{Z}_{i}(t ; z, \bar{z})=\bar{z}_{i}+\sum_{n=1}^{\infty} \frac{t^{-n}}{n} \widetilde{p}_{n}^{i}(z, \bar{z}) .
$$

One can get the solution (20) with $\widetilde{p}_{1}^{i}=-1 / 2 \epsilon^{i j} \partial_{\bar{z}_{j}} K$ and $\Omega=i / 2 \partial_{i} \bar{\partial}_{j} K d z^{i} \wedge d \bar{z}^{j}$.

The $t$-dependent Darboux coordinates $Z_{i}(t ; z, \bar{z})$ and $\widetilde{Z}_{i}(t ; z, \bar{z})$ correspond to holomorphic coordinates on two local charts, where the 2-form $\Psi(t)$ becomes the holomorphic $(2,0)$-form, of the dual projective twistor space $P^{*} T$ which may be viewed as a fiber bundle over $\mathbf{S}^{2}$ with 
a fiber being a hyper-Kähler manifold $\mathcal{M}_{4}$. Here we regard $t$ as a deformation parameter of complex structure on $\mathcal{M}_{4}$. We know from Eq.(19) that the Kähler form $\Omega$ is rank 4 and so can always serve as a symplectic form on both coordinate charts. As a result, two sets of coordinates at $t=0$ and $t=\infty$ are related to each other by a $t$-dependent canonical transformation given by $f_{i}(t ; Z(t))=t \widetilde{Z}_{i}(t)$ on an overlapping coordinate chart [15, 26]. In this way, the complex geometry of the twistor space $P^{*} T$ encodes all the information about the Kähler geometry of self-dual 4-manifolds $\mathcal{M}_{4}$ emergent from selfdual NC gauge fields. We refer the reader to [18] for more details about the twistor geometry.

From the above construction, we see that the information of self-dual NC gauge fields is encoded in the twistor space in the following way. The Bianchi identity $d F=0$ appears as the Kähler condition of a self-dual metric on $\mathcal{M}_{4}$ or as $d \Psi(t)=0$ in the twistor space. The self-duality equation (13) is realized as the Ricci flatness of $\mathcal{M}_{4}$ or as the nilpotentness (21) in the twistor space. In total, the self-dual NC gauge fields manifest themselves as a Ricci flat Kähler manifold $\mathcal{M}_{4}$ or as a holomorphic deformation of the twistor space. If turning off gauge fields, namely $F=0$, we simply arrive at a flat space, i.e., $\mathcal{M}_{4}=\mathbf{R}^{4}$ and $P^{*} T=\mathbf{R}^{4} \times \mathbf{S}^{2}$. Thus we should think of the twistor space as already incorporating the backreaction of NC instantons. This picture is remarkably similar to that in twistor string theory [17] as we discussed in Section 1.

\section{SELF-DUAL EINSTEIN GRAVITY FROM NC ELECTROMAGNETISM}

In this section we will use the background independent formulation of NC gauge theories [20, 28]. One can show using the SW map in [23, 24] for this case that

$$
\begin{aligned}
& \int d^{4} y(\widehat{F}-B)_{\mu \nu} \star(\widehat{F}-B)^{\mu \nu} \\
= & \int d^{4} x \sqrt{\operatorname{detg}} \mathrm{g}^{\mu \lambda} \mathrm{g}^{\sigma \nu} B_{\mu \nu} B_{\lambda \sigma}
\end{aligned}
$$

where $B_{\mu \nu}=\left(\theta^{-1}\right)_{\mu \nu}$. The identity (29) definitely shows that the fluctuations $\widehat{F}$ by NC photons around the background $B$ are mapped through the SW map to the fluctuations of geometry on commutative spacetime.

Before going into details, let us point out that the NC gauge theory and gravity correspondence may be understood as a large $N$ duality 2]. To see this picture, consider the NC description in [20, 28] where the action is expressed only in terms of manifestly covariant and background-independent variables:

$$
\begin{aligned}
& \frac{1}{4 G_{s}} \int d^{4} y(\widehat{F}-B)_{\mu \nu} \star(\widehat{F}-B)^{\mu \nu} \\
= & -\frac{\pi^{2}}{g_{s} \kappa^{2}} g_{\mu \lambda} g_{\nu \sigma} \operatorname{Tr}_{\mathcal{H}}\left[x^{\mu}, x^{\nu}\right]\left[x^{\lambda}, x^{\sigma}\right]
\end{aligned}
$$

where we made a replacement $\frac{1}{(2 \pi)^{2}} \int \frac{d^{4} y}{|\operatorname{Pf} \theta|} \leftrightarrow \operatorname{Tr}_{\mathcal{H}}$ using the Weyl-Moyal map. Here we used the following notation: $g_{\mu \nu}=$ constant closed string metric, $g_{s}\left(G_{s}\right)$ $=$ closed (open) string coupling constant, $\kappa=2 \pi \alpha^{\prime}$. The covariant, background-independent coordinates $x^{\mu}$ 12, 28] are defined by Eq.(4) and they are now operators acting on an infinite-dimensional, separable Hilbert space $\mathcal{H}$, which is the representation space of the Heisenberg algebra (11). The NC gauge symmetry in Eq. (30) then acts as unitary transformations on $\mathcal{H}$, i.e.,

$$
x^{\mu} \rightarrow x^{\prime \mu}=U x^{\mu} U^{\dagger} .
$$

This NC gauge symmetry $U_{\text {cpt }}(\mathcal{H})$ is so large that $U_{\text {cpt }}(\mathcal{H}) \supset U(N)(N \rightarrow \infty)$ [29]. In this sense the NC gauge theory in Eq. (30) is indeed a large $N$ gauge theory. Note that the second expression in Eq.(30) is a large $N$ version of the IKKT matrix model which describes the nonperturbative dynamics of type IIB string theory [30].

The map (5) in general defines an inner derivation of $\mathrm{NC} \star$-algebra which reduces to ordinary vector fields or Lie derivatives in the limit of slowly varying fields. Therefore we will identify the adjoint action of $D_{\mu}$ with respect to the star-product with vector fields $V_{\mu} \in T \mathcal{M}_{4}$ on some four manifold $\mathcal{M}_{4}$ as an emergent geometry. The identification is quite natural since the NC gauge fields $\widehat{A}_{\mu}(y)$ are in general arbitrary, so they generate arbitrary vector fields in $T \mathcal{M}_{4}$ according to the map (5) and $-i\left[B_{\mu \nu} x^{\nu}, f\right]_{\star}=\partial_{\mu} f$ when $\widehat{A}_{\mu}=0$. One can easily check that

$$
\begin{aligned}
& \left(\operatorname{ad}_{D_{\mu}} \operatorname{ad}_{D_{\nu}}-\operatorname{ad}_{D_{\nu}} \operatorname{ad}_{D_{\mu}}\right)[f] \\
= & -i \operatorname{ad}_{\left[D_{\mu}, D_{\nu}\right]_{\star}}[f] \approx\left[V_{\mu}, V_{\nu}\right][f]
\end{aligned}
$$

where the right-hand side is defined by the Lie bracket between vector fields in $T \mathcal{M}_{4}$.

Now let us look for an instanton solution of Eq. (30). Since the instanton is a Euclidean solution with a finite action, the instanton configuration should approach to a pure gauge at infinity. Our boundary condition is $\widehat{F}_{\mu \nu} \rightarrow 0$ at $|y| \rightarrow \infty$ as usual. Thus one has to remove the background part from the action (30), but with a background independent as well as gauge covariant way. There is a unique way to achieve this by defining the self-duality equation as follows

$$
\begin{aligned}
& \operatorname{ad}_{\left[D_{\mu}, D_{\nu}\right]_{\star}}= \pm \frac{1}{2} \varepsilon_{\mu \nu \lambda \sigma} \operatorname{ad}_{\left[D_{\lambda}, D_{\sigma}\right]_{\star}} \Leftrightarrow \\
& {\left[V_{\mu}, V_{\nu}\right]= \pm \frac{1}{2} \varepsilon_{\mu \nu \lambda \sigma}\left[V_{\lambda}, V_{\sigma}\right],}
\end{aligned}
$$

where we used Eq. (32). From the above definition, it is obvious that the constant part in $-i\left[D_{\mu}, D_{\nu}\right]_{\star}=\widehat{F}_{\mu \nu}-$ $B_{\mu \nu}$, i.e. $B_{\mu \nu}$, can be dropped and so the self-duality equation (33) is equivalent to Eq. (8).

It is obvious from Eq. (5) that the vector fields $V_{\mu}$ are divergence free, i.e. $\partial_{a} V_{\mu}^{a}=0$. In other words, they preserve a fixed volume form $\varepsilon_{4}$, i.e., $\mathcal{L}_{V_{\mu}} \varepsilon_{4}=0, \forall \mu$, where 
$\mathcal{L}_{V_{\mu}}$ is the Lie derivative along $V_{\mu}$. In addition, the vector fields $V_{\mu}$ form an orthonormal frame up to some conformal factor since $\varepsilon_{4}\left(V_{1}, V_{2}, V_{3}, V_{4}\right) \propto \operatorname{det}\left(\frac{\partial x}{\partial y}\right)$ corresponds to the Jacobian factor for the coordinate transformation, $y^{\mu} \rightarrow x^{\mu}(y)$, which is non-vanishing unless $F+B=0$. In summary, instanton configurations in NC spacetime (1D) are mapped to the volume preserving diffeomorphism, denoted as $S \operatorname{Diff}\left(\mathcal{M}_{4}\right)$, satisfying Eq. (33).

So we arrive at the result of Ashtekar et al. [13]. Their result is summarized as follows [31]. Let $\mathcal{M}_{4}$ be an oriented 4-manifold and let $V_{\mu}$ be vector fields on $\mathcal{M}_{4}$ forming an oriented basis for $T \mathcal{M}_{4}$. Then $V_{\mu}$ define a conformal structure $[\mathcal{G}]$ on $\mathcal{M}_{4}$. Suppose that $V_{\mu}$ preserve a volume form on $\mathcal{M}_{4}$ and satisfy the self-duality equation

$$
\left[V_{\mu}, V_{\nu}\right]= \pm \frac{1}{2} \varepsilon_{\mu \nu \lambda \sigma}\left[V_{\lambda}, V_{\sigma}\right] .
$$

Then $[\mathcal{G}]$ defines an (anti-)self-dual and Ricci-flat metric.

Since $V_{\mu} \in \operatorname{LSDiff}\left(\mathcal{M}_{4}\right)$, the Lie algebra of $\operatorname{SDiff}\left(\mathcal{M}_{4}\right)$, form an orthonormal frame up to a conformal factor, they define a metric on $\mathcal{M}_{4}$. Indeed $V_{\mu}^{a}$ in Eq.(15) can be related to an inverse vierbein or tetrad on $\mathcal{M}_{4}$. Thus the self-dual metric is given by [13]

$$
\mathcal{G}^{a b}=\operatorname{det} V^{-1} V_{\mu}^{a} V_{\nu}^{b} \delta^{\mu \nu} .
$$

Note that the metric (35) becomes flat if NC gauge fields are trivial, say, $\widehat{A}_{\mu}=0$ since $V_{\mu}^{a}=\delta_{\mu}^{a}$ in this case. In other words, the flat spacetime is emergent from the uniform condensation of gauge fields, i.e., $B_{\mu \nu}=(1 / \theta)_{\mu \nu}$, which defines the NC spacetime (1) as a vacuum. But Eq.(35) implies that the presence of nontrivial gauge fields in the $\mathrm{NC}$ spacetime deforms the vacuum manifold, i.e. flat $\mathbf{R}^{4}$, to a curved manifold. We here confirm again the same picture emergent in Section 2 that $\mathrm{NC}$ instantons deform $\mathbf{R}^{4}$ to a self-dual Einstein manifold.

Motivated by the similarity of Eq.(34) to the selfduality equation of Yang-Mills theory, Mason and Newman showed [14] that, if we have a reduced Yang-Mills theory where the gauge fields take values in the Lie algebra of $S \operatorname{Diff}\left(\mathcal{M}_{4}\right)$, which is exactly the case in Eq. (33), Yang-Mills instantons are actually equivalent to gravitational instantons. We just showed that this is the case for NC electromagnetism.

It is a priori not obvious that the self-dual electromagnetism in $\mathrm{NC}$ spacetime is equivalent to the self-dual Einstein gravity. Therefore it should be helpful to have explicit nontrivial examples to appreciate how the equivalence is achieved. It is not difficult to find explicit selfdual solutions from Eq.(34). For example, the GibbonsHawking metric [10] was given in [31] and the real heaven solution [1] was done in [32].

The Gibbons-Hawking metric [10] is a general class of self-dual, Ricci-flat metrics with the triholomorphic $U(1)$ symmetry which describes a particular class of ALE and ALF instantons. Let $\left(a_{i}, U\right), i=1,2,3$, are smooth real functions on $\mathbf{R}^{3}$ and define $V_{i}=-a_{i} \frac{\partial}{\partial \tau}+\frac{\partial}{\partial x^{i}}$ and $V_{4}=$ $U \frac{\partial}{\partial \tau}$, where $\tau$ parameterizes a circle and $\vec{x} \in \mathbf{R}^{3}$. Then one can easily check that $\left(V_{i}, V_{4}\right)$ are divergence-free, namely, they belong to LSDiff $\left(\mathcal{M}_{4}\right)$ and the Killing vector $\partial / \partial \tau$ generates the triholomorphic $U(1)$ symmetry. Eq.(34) then reduces to the equation $\nabla U+\nabla \times \vec{a}=0$ and the metric whose inverse is Eq.(35) is given by

$$
d s^{2}=U^{-1}(d \tau+\vec{a} \cdot d \vec{x})^{2}+U d \vec{x} \cdot d \vec{x} .
$$

The real heaven metric [1] describes four dimensional hyper-Kähler manifolds with a rotational Killing symmetry which is also completely determined by one real scalar field. The vector fields $V_{\mu}$ in this case are given by 32]

$$
\begin{aligned}
V_{1} & =\frac{\partial}{\partial x^{1}}-\partial_{2} \psi \frac{\partial}{\partial \tau} \\
V_{2} & =\frac{\partial}{\partial x^{2}}+\partial_{1} \psi \frac{\partial}{\partial \tau} \\
V_{3} & =e^{\psi / 2}\left(\sin \left(\frac{\tau}{2}\right) \frac{\partial}{\partial x^{3}}+\partial_{3} \psi \cos \left(\frac{\tau}{2}\right) \frac{\partial}{\partial \tau}\right) \\
V_{4} & =e^{\psi / 2}\left(\cos \left(\frac{\tau}{2}\right) \frac{\partial}{\partial x^{3}}-\partial_{3} \psi \sin \left(\frac{\tau}{2}\right) \frac{\partial}{\partial \tau}\right)
\end{aligned}
$$

where the rotational Killing vector is given by $c_{i} \partial_{i} \psi \partial / \partial \tau$ with constants $c_{i}(i=1,2)$ and the function $\psi$ is independent of $\tau$. Then it is easy to see that $V_{\mu} \in \operatorname{LSDiff}\left(\mathcal{M}_{4}\right)$. Eq.(34) is now equivalent to the three-dimensional continual Toda equation $\left(\partial_{1}^{2}+\partial_{2}^{2}\right) \psi+\partial_{3}^{2} e^{\psi}=0$ and the metric is determined by Eq. (35) as

$d s^{2}=\left(\partial_{3} \psi\right)^{-1}\left(d \tau+a^{i} d x^{i}\right)^{2}+\left(\partial_{3} \psi\right)\left(e^{\psi} d x^{i} d x^{i}+d x^{3} d x^{3}\right)$

where $a^{i}=\varepsilon^{i j} \partial_{j} \psi$.

Although we concretely derived the equivalence, Eq. (33), between self-dual NC electromagnetism and selfdual Einstein gravity, it is nevertheless very surprising that the real heaven (38) as well as the ALE and ALF instantons (36) are solutions of $\mathrm{NC}$ electromagnetism.

\section{DISCUSSION}

We have shown a remarkable picture implying that gravity can emerge from gauge fields living in NC spacetime. This striking picture about gravity is originated from the fact that the fluctuation of gauge fields in NC spacetime can be interpreted as that of spacetime geometry via the Darboux theorem. It turns out [2, 21] that a basic reason for the correspondence between $\mathrm{NC}$ gauge theory and gravity is that the NC spacetime (1) admits an extra symmetry, the so-called $\Lambda$-symmetry (or $B$-field transformation), which is as large as diffeomorphism symmetry. Through the Darboux theorem, the $\Lambda$-symmetry can be considered as a par with diffeomorphisms. This is the underlying reason why gravity can 
emerge from gauge fields living in NC spacetime. One can also see this picture through the SW map in Eq.(29).

Some remarks are in order.

(1) Recently, it was found 33] that NC field theory is invariant under the twisted Poincaré symmetry where the action of generators is now defined by the twisted coproduct in the deformed Hopf algebras. Especially the NC spacetime (11) appears as a twisted Poincaré invariant as well as gauge invariant (since $\theta^{\mu \nu}=(1 / B)^{\mu \nu}$ ) while it has been argued to break the usual Lorentz symmetry. This fact seems to be consistent with the picture of emergent gravity, as we observed below Eq. (35), where a flat spacetime is not a priori given but emergent from the algebra (11) defining a NC spacetime. According to the motto of emergent gravity declaring that a geometry is defined by an algebra, we interpret that the NC algebra (1) defines a flat spacetime as a vacuum geometry (i.e., without fluctuating fields) in the context of emergent geometry. If it is true, it implies that a flat spacetime as well as its Lorentz symmetry is emergent from the NC $\star$-algebra (1), which is twisted Poincaré invariant as well as gauge invariant. (See the Seiberg's paper in [1] for a related argument.) We think that the twisted Poincaré symmetry, especially the deformed Hopf algebra and quantum group structures, will be important to understand the correspondence between NC field theory and gravity since underlying symmetries are always an essential guide for physics. Incidentally, this symmetry plays a prominent role to construct NC gravity [34].

(2) The canonical structures, in particular, complex and Kähler structures, of the self-dual system (34), have been fully studied in a beautiful paper [15]. The arguments in [15] are essentially the same as ours leading to Eq.(26). It was also shown there how Plebański's heavenly equations [27] can be derived from Eq. (34) and also how Eq.34 manifests the structure of twistor space. Furthermore it can be shown [35] that Eq. (34) can be reduced to the $\operatorname{sdiff}\left(\Sigma_{g}\right)$ chiral field equations in two dimensions, where sdiff $\left(\Sigma_{g}\right)$ is the area preserving diffeomorphisms of a Riemann surface of genus $g$. All these properties are deeply related to several integrable structures. Thus it will be very interesting to clarify the integrable structures inherent in the self-dual NC electromagnetism, self-dual gravity and twistor spaces.

\section{Acknowledgements}

We would like to thank Chong-Sun Chu, Harald Dorn, Giuseppe Policastro, Mario Salizzoni and Alessandro Torrielli for helpful discussions. This work was supported by the Alexander von Humboldt Foundation.
* Electronic address: hsyang@kias.re.kr

[1] J. Maldacena, Scientific American, pages 56-63, November 2005; N. Seiberg, Emergent Spacetime, hep-th/0601234 G. T. Horowitz and J. Polchinski, Gauge/gravity duality, gr-qc/0602037

[2] H. S. Yang, Mod. Phys. Lett. A22 (2007) 1119.

[3] S. Weinberg and E. Witten, Phys. Lett. 96B (1980) 59.

[4] G. 't Hooft, Dimensional Reduction In Quantum Gravity, gr-qc/9310026 L. Susskind, J. Math. Phys. 36 (1995) 6377.

[5] J. M. Maldacena, Adv. Theor. Math. Phys. 2 (1998) 231; S. S. Gubser, I. R. Klebanov and A. M. Polyakov, Phys. Lett. B428 (1998) 105; E. Witten, Adv. Theor. Math. Phys. 2 (1998) 253.

[6] D. Gross, A. Hashimoto and N. Itzhaki, Adv. Theor. Math. Phys. 4 (2000) 893.

[7] V. O. Rivelles, Phys. Lett. B558 (2003) 191.

[8] M. Salizzoni, A. Torrielli and H. S. Yang, Phys. Lett. B634 (2006) 427.

[9] H. S. Yang and M. Salizzoni, Phys. Rev. Lett. 96 (2006) 201602.

[10] G. W. Gibbons and S. W. Hawking, Phys. Lett. 78B (1978) 430.

[11] C. P. Boyer and J. D. Finley, III, J. Math. Phys. 23 (1982) 1126.

[12] J. Madore, S. Schraml, P. Schupp and J. Wess, Eur. Phys. J. C16 (2000) 161.

[13] A. Ashtekar, T. Jabobson and L. Smolin, Commun. Math. Phys. 115 (1988) 631.

[14] L. J. Mason and E. T. Newman, Commun. Math. Phys. 121 (1989) 659.

[15] S. Chakravarty, L. Mason and E. T. Newman, J. Math. Phys. 32 (1991) 1458.

[16] N. J. Hitchin, A. Karlhede, U. Lindström and M. Roček, Commun. Math. Phys. 108 (1987) 535.

[17] S. A. Hartnoll and G. Policastro, Adv. Theor. Math. Phys. 10 (2006) 181.

[18] R. Penrose, Gen. Rel. Grav. 7 (1976) 31.

[19] N. Nekrasov and A. Schwarz, Commun. Math. Phys. 198 (1998) 689.

[20] N. Seiberg and E. Witten, J. High Energy Phys. 09 (1999) 032.

[21] H. S. Yang, Int. J. Mod. Phys. A24 (2009) 4473.

[22] B. Jurčo and P. Schupp, Eur. Phys. J. C14 (2000) 367; H. Liu, Nucl. Phys. B614 (2001) 305.

[23] R. Banerjee and H. S. Yang, Nucl. Phys. B708 (2005) 434.

[24] H. S. Yang, Mod. Phys. Lett. A21 (2006) 2637.

[25] S. W. Hawking, Phys. Lett. 60A (1977) 81; T. Eguchi, P. B. Gilkey and A. J. Hanson, Phys. Rep. 66 (1980) 213.

[26] H. Ooguri and C. Vafa, Nucl. Phys. B361 (1991) 469.

[27] J. F. Plebański, J. Math. Phys. 16 (1975) 2395.

[28] N. Seiberg, J. High Energy Phys. 09 (2000) 003.

[29] J. A. Harvey, Topology of the Gauge Group in Noncommutative Gauge Theory, hep-th/0105242

[30] N. Ishibashi, H. Kawai, Y. Kitazawa and A. Tsuchiya, Nucl. Phys. B498 (1997) 467.

[31] D. D. Joyce, Duke Math. J. 77 (1995) 519.

[32] T. Ootsuka, S. Miyagi, Y. Yasui and S. Zeze, Class. Quant. Grav. 16 (1999) 1035.

[33] M. Chaichian, P. Kulish, K. Nishijima and A. Ture- 
anu, Phys. Lett. B604 (2004) 98; M. Chaichian, P. Presnajder and A. Tureanu, Phys. Rev. Lett. 94 (2005) 151602; J. Wess, Deformed Coordinate Spaces: Derivatives, hep-th/0408080

[34] P. Aschieri, C. Blohmann, M. Dimitrijevic, F. Meyer, P.
Schupp and J. Wess, Class. Quant. Grav. 22 (2005) 3511; P. Aschieri, M. Dimitrijevic, F. Meyer and J. Wess, Class. Quant. Grav. 23 (2006) 1883.

[35] V. Husain, Phys. Rev. Lett. 72 (1994) 800. 